\title{
Efeito do uso do traje de neoprene sobre variáveis técnicas, fisiológicas e perceptivas de nadadores
}

CDD. 20.ed. 797.2

\begin{tabular}{r|l} 
Karini Borges dos SANTOS* & *Universidade Federal \\
Paulo Cesar Barauce BENTO* & do Paraná. \\
André Luiz Félix RODACKI* &
\end{tabular}

\begin{abstract}
Resumo
Ao contrário do que ocorre em provas de piscina, competições em águas abertas estão sujeitas as condições ambientais, sendo uma delas as baixas temperaturas. Em determinadas circunstâncias é permitido o uso de roupas especiais para evitar hipotermia. 0 objetivo do estudo foi verificar os efeitos do uso da roupa de neoprene em um grupo composto por triatletas e nadadores, comparado ao uso de vestimentas convencionais (sunga) sobre variáveis cinemáticas e psicofisiológicas do nado. Participaram 20 homens (12 triatletas e oito nadadores) de idade $22,0 \pm 6,6$ anos com desempenhos que correspondem a $75 \pm$ $7,7 \%$ do melhor tempo brasileiro na prova de $400 \mathrm{~m}$. Os atletas realizaram duas repetições máximas e duas submáximas de $400 \mathrm{~m}$ em nado "crawl", com e sem o uso da roupa de neoprene. Foram comparadas a velocidade média (VM), comprimento de braçada (CB), frequência de braçada (FB), índice de nado (IN), percepção subjetiva de esforço (PSE), frequência cardíaca (FC), e concentração de lactato sanguíneo (LAC). Um conjunto de ANOVAs com medidas repetidas do tipo "two-way" foi aplicado. Quando diferenças foram encontradas o teste de Tukey foi empregado. Com o traje de neoprene, em máxima intensidade, o tempo para nadar a distância foi 6,4\% menor, com manutenção da $F B$ e aumento da $C B$, as variáveis psicofisiológicas não diferiram estatisticamente. Em esforço submáximo, o uso do traje de neoprene resultou em menor FB, maior $C B$, maior IN e em menores valores de $F C$, LAC e PSE $(p<0,05)$. 0 uso do traje proporcionou melhoria do desempenho nos aspectos biomecânicos, fisiológicos e perceptivos e que o aumento da VM em esforço máximo não depende exclusivamente de alterações na FB e CB. Possivelmente, incrementos nos parâmetros associados ao nado pode ter melhorado a eficiência mecânica do movimento, a qual pode ter provido uma economia de movimento que resultou em melhor desempenho.
\end{abstract}

UnItERMos: Traje especial; Natação; Desempenho.

\section{Introdução}

As provas de natação realizadas em águas abertas tiveram sua popularidade aumentada a partir da inclusão do triatlo e das maratonas aquáticas no programa de provas dos Jogos Olímpicos. Ao contrário do que ocorre em provas de piscina, nas provas em águas abertas os competidores estão sujeitos a diferentes condiçóes ambientais que envolvem correntes, turbulência e principalmente baixas temperaturas da água. Para minimizar os efeitos das baixas temperaturas os competidores têm utilizado roupas especiais de neoprene. Para a categoria de elite, o traje de neoprene é permitido em temperaturas abaixo de $20^{\circ} \mathrm{C}$ (Confederação Brasileira de Triathlon, 2010). O traje de neoprene começou a ser utilizado a partir do exemplo dos surfistas e mergulhadores que desejavam minimizar os problemas térmicos de perda de calor corporal - o risco de hipotermia (DE LUCAS, BALIKIAN, Neiva, Greco \& Denadai, 2000).

Além de isolante térmico, alguns estudos têm demonstrado que o traje de neoprene pode melhorar o rendimento durante as provas de natação (CARVALHO, Viana, Parra, Copetti, Porto, Rodrigues, Félix, Moraes \& Alves Júnior, 2006; Cordain \& Kopriva, 1991; LOWDON, MCKENZIE \& Ridge, 1992; PARSONS \& Day, 1986; Toussaint, Bruinic, Coster, De LoOze \& Rossem, 1989). O desempenho na natação 
está em grande parte determinado pela capacidade de gerar forças propulsivas e de reduzir as forças que se opõe ao deslocamento (forças resistivas). A redução da densidade corporal causada pelo traje de neoprene influencia a flutuação que reduz a resistência da água (Cordain \& Kopriva, 1991). Além disso, essa melhor flutuação permite um melhor posicionamento do corpo e influencia positivamente alguns aspectos hidrodinâmicos associados à resistência da forma do corpo ao avanço (MAGLISCHO, 2003). Assim, a maior parte da energia despendida pelo nadador pode ser mais bem aproveitada para a propulsão, aumentando sua eficiência mecânica (CAPUTO, Fernandes, Oliveira, Sérgio \& Coelho, 2006; Maglischo, 2003).

Os estudos realizados com o objetivo de verificar os efeitos do uso do traje de neoprene no rendimento do nadador têm comparado a velocidade ou tempo para nadar determinada distância em máximo esforço (Cordain \& Kopriva, 1991; De Lucas et al., 2000; Toussaint et al., 1989). Além disso, variáveis de composição corporal (CoRDAin \& Kopriva, 1991), arrasto propulsivo (De Lucas, Balikian, Neiva, Greco, \& Denadai, 2000; Toussaint et al., 1989) e a velocidade de nado correspondente ao limiar anaeróbio (De LuCAS et al., 2000) têm sido analisadas. Há consenso nesses estudos que o traje de neoprene proporciona aumento da velocidade, embora não haja concordância em relação à magnitude destas alterações, onde os resultados apontam variações entre $3 \%$ e 10\% (Cordain \& Kopriva, 1991; LowdOn, McKenZIE \& Ridge, 1992, respectivamente).

Em condiçôes normais de nado (sem o traje de neoprene), o aumento da velocidade na natação ocorre em função da interação (aumento/diminuição) entre o comprimento da braçada (CB) e frequência de braçadas (FB) (Craig \& Pendergast, 1979), onde valores máximos ou mínimos desses parâmetros podem gerar tempos mais lentos. Sabe-se que uma combinação ótima entre esses fatores resulta em velocidades mais elevadas (CAputo et al., 2006; Castro, Guimarães, Moré, LammerhirT \& MarQues, 2005; SMith, DaVID, Norris, Stephen, Hogg \& Johnm, 2002). Devido à relevância destes fatores, a procura de indicadores correlacionados com a mecânica do nado tornou-se frequente para elevados desempenhos. Assim, o índice de nado (IN; produto entre a velocidade média de nado e o $\mathrm{CB}$ ) tem sido empregado como um dos parâmetros associados a uma técnica de movimento mais apurada (CAPUTO et al., 2006). No entanto, o comportamento dessas variáveis $(\mathrm{CB}, \mathrm{FB}$ e IN) ainda não foi investigado quando o aumento da velocidade decorre das supostas vantagens proporcionadas pela utilização do traje de neoprene. Em adição, essas variáveis não foram analisadas quando os atletas realizam o teste com e sem o traje de neoprene em uma intensidade relativa semelhante. A avaliação do desempenho em condições submáximas é importante, por permitir comparaçôes da influência do traje sobre um conjunto de parâmetros do nado, independente de variaçōes da velocidade. Além disso, muitos esforços empregados no treinamento e ao longo de algumas provas de natação não são máximos e podem ser influenciados pela vestimenta.

Assim, é relevante comparar o comportamento de parâmetros fisiológicos em diferentes esforços (máximo e submáximo), visto que se esperam melhores respostas cardiovasculares e metabólicas decorrentes do uso do traje. Tais benefícios também podem ser refletidos sobre a percepção do esforço realizado, a PSE é considerada um bom indicador da intensidade relativa de esforço na natação (MAGLISCHO, 2003), além disso, correlações elevadas foram encontradas entre a PSE, a frequência cardíaca (FC) e a concentração de lactato sanguíneo (LAC) em natação estacionária em intensidades submáximas (Ueda \& KurokaWa, 1995). No entanto, não são conhecidos estudos que tenham associado variáveis que descrevam aspectos técnicos, fisiológicos e perceptivos em condiçôes experimentais em que se manipula o tipo de vestimenta durante provas de natação em diferentes intensidades.

Desta forma, o objetivo do presente estudo foi comparar a influência da utilização do traje de neoprene sobre aspectos técnicos (velocidade média - VM; comprimento - $\mathrm{CB}$ e frequência - FB de braçadas; e o índice de nado - IN), fisiológicos (frequência cardíaca - FC; concentração de lactato LAC) e perceptivos do esforço (percepção subjetiva de esforço - PSE) em diferentes intensidades de nado (máxima e submáxima). 


\section{Métodos}

\section{Participantes}

Participaram do estudo 20 atletas do sexo masculino (12 triatletas e oito nadadores; $22,0 \pm 6,6$ anos; $68,2 \pm 8,2 \mathrm{~kg}$ e $1,76 \pm 0,06 \mathrm{~m})$, com experiência competitiva mínima de dois anos e que treinavam natação pelo menos cinco dias por semana, com desempenhos que correspondem a $75 \pm 7,7 \%$ e 72 $\pm 8,0 \%$ dos melhores tempos brasileiro e mundial, respectivamente, na prova de $400 \mathrm{~m}$.

Antes do início do estudo, os sujeitos foram informados dos procedimentos da pesquisa e assinaram termo de consentimento livre e esclarecido. O protocolo de pesquisa foi aprovado pelo Comitê de Ética em Pesquisa com Seres Humanos do Setor de Saúde da Universidade Federal do Paraná (n $2431.0 .000 .091-08)$.

\section{Procedimentos}

Cada participante realizou quatro simulaçōes da prova de $400 \mathrm{~m}$ em uma piscina de $25 \mathrm{~m}$, coberta e aquecida com água controlada em $29^{\circ} \mathrm{C}$. Duas dessas simulações foram realizadas utilizando um traje de natação convencional (sunga de lycra) e uma roupa de neoprene (modelo "full", espessura de 1,5 $\mathrm{mm}$, Mormaii, Brasil) em intensidade máxima, as quais foram denominadas LYCMAX e NEOMAX, respectivamente. $\mathrm{O}$ modelo empregado permitiu cobertura da superfície corporal até os punhos e tornozelos. As outras duas simulações foram realizadas com as mesmas condiçōes experimentais de traje, porém em intensidade submáxima e denominadas LYCSUB e NEOSUB, respectivamente. Logo, o estudo contou com quatro condiçóes experimentais (traje [LYC e NEO] e intensidade [MAX e SUB]).

As simulações que envolveram variação de intensidade (MAX e SUB) foram sempre realizadas numa mesma sessão e tiveram um intervalo de 30 a 40 minutos. Durante este período de intervalo os atletas foram orientados a nadar em baixa intensidade por 20 minutos para garantir uma adequada remoção do LAC. O emprego de um protocolo fracionado ao longo de outros dias não foi possível pela demanda de treinamento dos atletas. Alguns estudos têm demonstrado que tal período é suficiente para a redução sérica do LAC (MACARDLE, 2003). Um período entre $48 \mathrm{~h}$ e duas semanas foi empregado entre os testes das condições experimentais de vestimenta (LYC e NEO). Um período de aquecimento de 10 minutos de nado livre em baixa intensidade foi permitido no início de cada sessão. Nos testes máximos, os sujeitos foram instruídos a realizar seu melhor desempenho, a condição LYCMAX foi utilizada como referência para a determinação dos testes em intensidade submáxima. O controle da velocidade nos testes submáximos foi feito por intermédio de plaquetas indicativas de tempo que foram fornecidas a cada $50 \mathrm{~m}$. Tal informação permitiu orientar o ritmo do nado a fim de controlar a intensidade da prova que foi ajustada para aproximadamente $90 \%$ da intensidade máxima daquela obtida do nado máximo com lycra (LYCMAX).

Para determinar o desempenho do nado, um conjunto de variáveis cinemáticas foi determinado e empregado para caracterizar os componentes associados à técnica do nado. As variáveis cinemáticas compreenderam a frequência (FB) e o comprimento das braçadas $(\mathrm{CB})$, a velocidade média da prova $(\mathrm{VM})$ e o índice de nado (IN). O CB corresponde à distância percorrida pelo nadador em cada braçada, enquanto que a FB foi determinada pelo número de ciclos de braçada (um ciclo corresponde a duas entradas consecutivas da mesma mão na água) no intervalo de um minuto e foi calculada com o auxílio de um cronômetro manual sobre a média de três ciclos de braçadas realizados na regiáo central da piscina (60 segundos dividido pelo tempo médio de três ciclos). Assim, os resultados dessas três braçadas foram extrapolados a fim de expressá-los em um minuto. A VM foi calculada pelo quociente entre a distância percorrida (400 m) e o tempo necessário para concluir a prova. Considerando que a VM é determinada pelo produto entre a $\mathrm{FB}$ e o $\mathrm{CB}$, o $\mathrm{CB}$ foi estimado pela razão entre a VM e a FB (CAPUTO, LUCAS, GreCo \& DenadaI, 2000). Reconhece-se que a VM expressa a velocidade aplicada na prova e não contempla apenas a velocidade de nado propriamente dita, uma vez que considera as demais fases do nado (saída, viradas e fases submersas). Todavia, a influência de tal negligência tem sido calculada e apresenta uma super estimativa de 3\% a 2,6\% nas distâncias de 100 e 1500 m, respectivamente (Craig \& Pendergast, 1979). O IN tem sido empregado pela literatura como uma forma de determinar o desempenho técnico nas provas de natação e foi determinado pelo produto do $\mathrm{CB}$ pela VM (CAPUTO et al., 2000).

Para avaliação da percepção subjetiva de esforço (PSE), os participantes foram orientados a indicar imediatamente ao final da prova o nível subjetivo de esforço realizado, qualificando a dificuldade de sua realização. Para essa finalidade a escala de Borg 
de seis a 20 foi empregada (BORG, 2000). A FC foi determinada por meio de um frequencímetro (Polar, modelo F1 - Finlândia) durante os primeiros 5 $s$ após o final da prova. A concentração de lactato sanguíneo (LAC) foi quantificada através da análise sanguínea de cinco a 25 microlitros (Acusport ${ }^{\circledR}$ Boehring Mannheim), extraída da extremidade distal do dedo indicador, ao final do primeiro minuto após cada prova (MAgLisCHO, 2003).

\section{Análise estatística}

Os testes de Shapiro-Wilk e Levene foram aplicados e confirmaram a normalidade e homogeneidade dos dados, que foram tratados por estatística descritiva padrão (média e desvio padrão).

\section{Resultados}

Os procedimentos de controle da intensidade de esforço empregados no estudo para a prova de 400 $\mathrm{m}$ foram bem sucedidos. A VM no teste máximo empregado como referência para as comparações (LYCMAX) entre as condições não apresentou diferenças significativas $(\mathrm{p}<0,05)$ entre triatletas e nadadores e permitiu agrupar os atletas de diferentes modalidades esportivas. Em esforço submáximo, as intensidades relativas calculadas com base no tempo do nado com traje de lycra em máxima velocidade (100\%) foram de $91,1 \%$ vs $91,5 \%$ para a lycra e neoprene $(\mathrm{p}>0,05)$, respectivamente. É necessário destacar que os valores máximos (100\%) foram empregados como referência para permitir comparações entre as variáveis do estudo.

O tempo do nado "crawl" com o uso da roupa de neoprene em esforço máximo foi 6,4\% mais baixo ( $\mathrm{p}$ $<0,05)$ do que aquele obtido com traje tradicional de lycra (277 s vs 295 s). Em consequência, a VM também foi maior $(\mathrm{p}<0,05)$ quando o traje de neoprene foi utilizado. Os atletas apresentaram maior CB (percorreram maior distância por braçadas) e obtiveram maiores valores para o IN (produto da VM pelo $\mathrm{CB})(\mathrm{p}<0,05)$ em condição máxima, enquanto a $\mathrm{FB}$ não diferiu entre as
Inicialmente, um teste t pareado foi aplicado para comparar as diferenças entre as intensidades dos nados (MAX e SUB) em cada um dos trajes testados (LYC e NEO). Um número de ANOVAs com medidas repetidas do tipo "two-way" foi aplicada para identificar as diferenças entre as variáveis independentes que corresponderam às condições experimentais de vestimenta (LYC e NEO) e intensidade (MAX e SUB). As variáveis dependentes compreenderam as variáveis cinemáticas $(\mathrm{CB}, \mathrm{FB}$, IN e VM), fisiológicas (FC e LAC) e perceptivas (PSE). O teste de Tukey foi empregado para determinar onde as diferenças ocorreram. Os testes foram calculados em um pacote estatístico (Statistica, versão 7.0) e tiveram seu nível de significância fixado em $\mathrm{p}<0,05$.

condiçōes $(\mathrm{p}>0,05)$. Os aspectos psicofisiológicos não apresentaram diferenças para nenhuma das variáveis em intensidade de esforço máximo. A TABELA 1 apresenta os resultados para as variáveis cinemáticas e psicofiológicas nas condições máxima e submáxima de esforço em função dos diferentes trajes empregados.

Em intensidade submáxima, o desempenho correspondeu a aproximadamente $91 \%$ do máximo em ambas as condiçōes experimentais, ou seja, o efeito da velocidade de nado foi neutralizado ao se impor uma intensidade relativa equivalente entre as provas realizadas com e sem o traje de neoprene (LYCSUB e NEOSUB). Ao utilizar o traje de neoprene, os atletas apresentaram menor $\mathrm{FB}(\mathrm{p}<$ $0,05)$, maior $\mathrm{CB}(\mathrm{p}<0,05)$ e, consequentemente, apresentaram melhores INs $(\mathrm{p}<0,05)$. Foi possível verificar efeitos psicofisiológicos positivos decorrentes do uso do traje de neoprene, posto que os atletas realizaram o mesmo esforço relativo com uma menor percepção subjetiva do esforço empregado $(p<0,05)$. A FC foi menor $(p<0,05)$ e a concentração de lactato sanguíeno mais baixa ( $\mathrm{p}$ $<0,05)$ no nado com roupa de neoprene (NEO) em comparação à vestimenta tradicional (LYC). 
TABELA 1 - Média ( \pm desvio padrão) dos parametros cinemáticos e psicofisiológicos do nado em teste de esforço máximo (MAX) e submáximo (SUB) com traje de lycra (LYC) e neoprene (NEO).

\begin{tabular}{lcccc}
\hline \multicolumn{1}{c}{ Variáveis } & LYCMAX & NEOMAX & LYCSUB & NEOSUB \\
\hline VM (m/s) & $1,36 \pm 0,07$ & $1,44 \pm 0,08^{*}$ & $1,23 \pm 0,06$ & $1,24 \pm 0,06$ \\
FB (ciclos/min) & $36,40 \pm 4,54$ & $36,35 \pm 4,52$ & $30,65 \pm 3,70$ & $28,20 \pm 3,70^{*}$ \\
CB $(\mathrm{m})$ & $2,27 \pm 0,26$ & $2,39 \pm 0,27^{*}$ & $2,46 \pm 0,28$ & $2,69 \pm 0,28^{*}$ \\
IN & $3,09 \pm 0,41$ & $3,51 \pm 0,41^{*}$ & $3,05 \pm 0,41$ & $3,34 \pm 0,49^{*}$ \\
PSE & $17,1 \pm 1,71$ & $17,1 \pm 1,59$ & $12,2 \pm 2,12$ & $10,75 \pm 1,88^{*}$ \\
FC (bpm) & $172 \pm 11,13$ & $169 \pm 17,14$ & $149 \pm 17,96$ & $134 \pm 19,45^{*}$ \\
LAC $(\mathrm{mml} / \mathrm{l})$ & $8,8 \pm 2,22$ & $8,8 \pm 2,79$ & $5,3 \pm 1,65$ & $3,8 \pm 1,21^{*}$ \\
\hline
\end{tabular}

Vm = Velocidade Média; $\mathrm{FB}=$ Frequencia de braçada; $\mathrm{CB}=$ Comprimento de braçada;

IN = Índice médio de nado;

$\mathrm{FC}=$ Frequencia cardiaca;

PSE = Percepção subjetiva de esforço; $\mathrm{LAC}=$ Lactato sanguineo (LAC); ${ }^{*} p<0,05$.

\section{Discussão}

O objetivo do presente estudo foi investigar a influência da utilização roupa de neoprene sobre um conjunto de variáveis cinemáticas e fisiológicas, além de verificar a percepção do esforço realizado pelo atleta em duas intensidades de nado (máxima e submáxima). Os estudos que compararam as duas condições de nado (com e sem o uso do traje de neoprene) verificaram que a roupa de neoprene proporcionou o aumento da velocidade de nado em intensidade máxima entre 3\% (CORDAIN \& KoprIVA, 1991) e 10\% (Lowdon, McKenzie \& Ridge, 1992).

No presente estudo, o tempo necessário para nadar os $400 \mathrm{~m}$ com o uso do traje de neoprene foi $6,4 \%$ menor comparado ao traje tradicional, o que representou uma diferença de 18 s para councluir a prova. Estes resultados estão próximos as diferenças encontradas por CARVALHO et al. (2006) que avaliaram triatletas e verificaram uma diferença média de $7,4 \%$ no tempo total de triatletas com menor eficiência mecânica e apenas $4,7 \%$ quando considerados os tecnicamente mais hábeis. No entanto, tais comparações requerem cautela uma vez que o teste foi realizado em uma distancia de 1500 m. CORDAIN e KoprIva (1991) encontraram uma redução correspondente a 4,96\% em uma prova de 400 m e 3,23\% em uma prova de 1500 $\mathrm{m}$. Diferenças mais expressivas foram apontadas por Lowdon, McKenziE e Ridge (1992), no qual a variação de desempenho foi de 10\%. As discrepâncias entre estudos podem ser atribuídas ao nível técnico entre os nadadores, sendo que os que possuíam melhor desempenho experimentaram menores diferenças quando utilizaram o neoprene. Diferenças na variabilidade em função da distância também podem ter influenciado, visto as variaçôes observadas em distâncias maiores tendem a ser menores (p.ex. 400 vs 1500) (CORDAIN
\& Kopriva, 1991). Outros fatores como diferenças na composição corporal também podem auxiliar a explicar tais variaçôes, posto que atletas com maior massa corporal magra possuem incrementos maiores na performance (CORDAIN \& KopRIVA, 1991).

Ao comparar as variáveis cinemáticas em esforço máximo, a VM foi maior com o uso do neoprene. Os atletas puderam aumentar a VM sem alterar a FB. Parece que tais aumentos foram obtidos por aumentos no CB. Em geral, aumentos na velocidade de nado são conseguidos por incrementos na $\mathrm{FB}$, os quais são acompanhados por redução no CB (CRAIG \& Pendergast, 1979; Craig, Skehan, PawelczyK \& BoOMer, 1985). No presente estudo essa tendência não foi confirmada quando os atletas nadaram como a roupa de neoprene. Provavelmente, as melhorias sobre a flutuação dos participantes (CORDAIN \& KoPRIVA, 1991) tenham permitido a adoção de uma posição mais hidrodinâmica que tenha conduzido a uma redução nas forças de arrasto. Assim, parte da energia despendida para vencer a resistência ao avanço pode ser utilizada para uma propulsão mais efetiva a cada ciclo de braçadas (MAGLISCHO, 2003).

Em esforço submáximo, o efeito da VM sobre os demais parâmetros cinemáticos pode ser neutralizado, permitindo comparações entre as condições experimentais, sem efeito da intensidade do esforço. Nas condições submáximas, os atletas apresentaram menor FB, maior CB e maior IN quando utilizaram o traje de neoprene. Similarmente ao apontado em esforços máximos, a melhor flutuação induzida pelo traje de neoprene pode ter influenciado a performance e produzido melhores resultados.

Ao melhorar a flutuação e reduzir as forças resistivas, provavelmente os atletas puderam aplicar 
força propulsiva mais efetivamente e melhorar o desempenho no nado. Tal argumento está em consonância com a afirmação de que os atletas devem apresentar uma boa técnica de nado aliada a uma elevada capacidade de produzir altos níveis de energia para sustentar esforços repetitivos por longo periodo (CAPUTO et al., 2006). A similaridade nos parâmetros de FC e LAC entre as condições experimentais em esforço máximo demonstra que os esforços foram similares e atingiram elevado índice de esforço percebido. No entanto, ao comparar esses indicadores em velocidade submáxima, novamente pode-se observar as vantagens proporcionadas pelo uso do traje de neoprene. Em esforço relativo semelhante, os atletas tiveram menor percepção do esforço realizado, confirmados pelos índices objetivos como menor FC e menor concentração de LAC. Logo, houve menor sensação de esforço e menor sobrecarga cardiovascular e metabólica para o mesmo esforço relativo (91\% do máximo).

\section{Conclusões}

O presente estudo analisou o desempenho de atletas com experiência em natação em duas intensidades (máxima e submáxima) com o uso do traje tradicional de lycra e neoprene. Em esforços máximos, os atletas foram capazes de nadar mais rápido quando utilizaram o traje de neoprene, sem aumentar a FB. A maior VM foi acompanhada por incremento do CB e do IN, o que sugere efeito do traje sobre a flutuação, posição corporal e, portanto, redução do arrasto propulsivo.

Para realizar um mesmo esforço relativo (90\% do LYCMAX), com o uso do neoprene os atletas realizaram menor freqüência de braçadas, percorreram maior distância a cada ciclo de braçadas e apresentaram um melhor índice de nado que demonstra maior eficiência mecânica. Não houve diferença para a resposta cardiovascular e metabólica no teste máximo, o que demonstra um esforço de alta intensidade nas duas condições de teste. $\mathrm{O}$ mesmo ocorreu em relação ao esforço percebido para o teste máximo.

Nos testes submáximos, a sobrecarga cardiovascular, metabólica e o esforço percebido foram menores quando utilizado o traje de neoprene. Estes achados permitem atribuir e quantificar de forma mais objetiva os efeitos proporcionados pelo uso da roupa de neoprene. Portanto, o uso do traje nas provas apresenta uma vantagem quando comparado à condição sem traje e deve ser empregado sempre que os regulamentos das competições permitam.

\section{Abstract}

Effects of a neoprene suit over technical, physiological and perceptive variables of swimmers

In open water swimming competitions, athletes are prone to environmental conditions and are frequently exposed to low temperatures, in contrast to what occurs in indoor competitions. In some circumstances the use of special swimming suits is allowed to avoid hypothermia. The aim of this study was to verify the effects of the use of a neoprene swimming suit in comparison to a conventional swimming suit on a number of cinematic and psychophysiological variables. Twenty athletes experienced in swimming competitions ( 12 tri-athletes and 8 swimmers; $22.0 \pm 6.6$ years-old), whose the performance was $75 \pm$ $7.7 \%$ of the National record. Athletes performed two maximal and two sub-maximal $400 \mathrm{~m}$ crawl simulated competition with a whole body neoprene swimming suit and with a conventional swimming suit. Mean speed (VM) stroke length (CB), stroke frequency (FB), swimming index (IN), rate of perceived effort (PSE), heart rate, and blood lactate concentration (LAC) were compared between conditions. The time to perform maximal trials with the use of the neoprene swimming suit was $6.4 \%$ shorter than when wearing the traditional swimming suit. FB and the psychophysiological variables remained unchanged, while CB increased in response to the use of the neoprene swimming suit. The use of the neoprene swimming suit in sub-maximal trials provided smaller FB, FC, LAC and PSE and larger CB and IN in comparison to the use of the traditional swimming suit. The results indicated that the neoprene swimming suit increases performance in biomechanical, physiological and perceptive aspects. The VM increase in maximal efforts 
does not depend exclusively on changes in FB and CB. Possibly, increments of the parameters related to the swimming may have improved the mechanical efficiency of the movement, which may have provided a movement economy that resulted in a better performance.

UNITERMS: Special swimming suits; Swimming; Performance.

\section{Referências}

BORG, G. Escalas de Borg para dor e esforço percebido. São Paulo: Manole, 2000.

CAPUTO, F.; FERNANDES, M.; OLIVEIRA, M. D.; SÉRGIO, B.; COELHO, C. Fatores intrínsecos do custo energético da locomoção durante a natação. Revista Brasileira de Medicina, São Paulo, v.12, p.399-404, 2006.

CAPUTO, F.; LUCAS, R.D.; GRECO, C.C.; DENADAI, B.S. Características da braçada em diferentes distâncias no estilo crawl e correlaçôes com a performance. Revista Brasileira de Ciência e Movimento, Brasília, v.8, n.3, p.7-13, 2000. CARVALHO, A.L.; VIANA, R.M.; PARRA, R.; COPETTI, L.R.; PORTO, P.A.D.C.; RODRIGUES, L.C.; FÉLIX, D.M.; MORAES, C.G.; ALVES JÚNIOR, J.M.M. Influência da utilização da roupa de neoprene sobre a performance do triatleta. Revista de Educação Física, Rio de Janeiro, n.135, p.22-7, 2006.

CASTRO, F.A.S.; GUIMARÂES, A.C.; MORÉ, F.C.; LAMMERHIRT, H.M.; MARQUES, A.C. Cinemática do nado crawl sob diferentes intensidades e condições de respiração de nadadores e triatletas. Revista Brasileira de Educação Física e Esporte, São Paulo, v.19, n.3, p.223-32, 2005.

CONFEDERAÇÃO BRASILEIRA DE TRIATLON. Manual de regras da Confederação Brasileira de Triathlon. Disponível em: <http://www.cbtri.org.br/docs/RegrasdeUniforme2010.pdf>. Acesso em: 24 nov. 2010.

CORDAIN, L.; KOPRIVA, R. Wetsuits, body density and swimming performance. British Journal of Sports Medicine, London, v.25, p.31-3, 1991.

CRAIG, A.B.; PENDERGAST, D.R. Relationships of stroke rate, distance per stroke, and velocity in competitive swimming. Medicine and Science in Sports and Exercise, Madison, v.11, n.3, p.278-83, 1979.

CRAIG, A.B.; SKEHAN, P.L.; PAWELCZYK, J.A.; BOOMER, W.L. Velocity, stroke rate, and distance per stroke during elite swimming competition. Medicine and Science in Sports and Exercise, Madison, v.17, n.6, p.625-34, 1985.

DE LUCAS, R.D.; BALIKIAN, P.; NEIVA, C.M.; GRECO, C.C.; DENADAI, S.B. The effects of wet suits on physiological and biomechanical indices during swimming methods and procedures. Journal of Science and Medicine in Sport, Belconen, v.3, n.1, p.1-8, 2000.

LOWDON, B.J.; McKENZIE, D.; RIDGE, B.R. Effects of clothing and water temperature on swim performance. Australian Jounal of Science and Medicine in Sports, Belconen, v.24, p.33-38, 1992.

MacARDLE, W.D. Fisiologia do xercício, energia nutrição e desempenho humano. Rio de Janeiro: Guanabara Koogan, 2003. MAGLISCHO, E.W. Swimming fastest. Champaign: Human Kinetics, 2003.

PARSONS, L.; DAY, S.J. Do wet suit effect swimming speed? British Journal of Sports Medicine, London, v.20, p.128-31, 1986. SMITH, D.J.; NORRIS, S.; HOGG, J.M. Performance evaluation of swimmers. Sports Medicine, Auckland, v.32, p.540-54, 2002.

TOUSSAINT, H.M.; BRUINIC, L.; COSTER, R.; DE LOOZE, M.; ROSSEM, B.V. Effect of a triathlon wet suit on drag during swimming. Medicine and Science in Sports and Exercise, Madison, v.21, p.325-8, 1989.

UEDA, T.; KUROKAWA, T. Relationships between perceived exertion and physiological variables during swimming. International Journal of Sports Medicine, Stuttgart, v.6, p.385-9, 1995.

ENDEREÇO

André Luiz Felix Rodacki Centro de Estudos do Comportamento Motor Departamento de Educação Física Universidade Federal do Paraná R. Coração de Maria, 92

80215-370 - Curitiba - PR - BRASIL e-mail: rodacki@ufpr.br
Recebido para publicação: 02/ 08/2010

Aceito: 04/ 02/2011 